the wall and moves outward. Professor L. Howarth has mentioned to me that he is working on this more complex aspect of the problem. [Ref. added Oct. 1, 1951: L. Howarth, Some aspects of Rayleigh's problem for a compressible fluid, Q. Jour. Mech. and Appl. Math., 4, pt. 2, 157 (1951)].

\title{
REFERENCES
}

(1) H. W. Emmons, Note on aerodynamic heating, Q. Appl. Math., 8, 402 (1951).

(2) Lord Rayleigh, On the motion of solid bodies through viscous liquid, Phil. Mag., (6) 21, 697 (1911) [Scientific Papers, Vol. 6, Cambridge University Press, 1920, p. 29].

(3) Th. v. Kármán, The problem of resistance in compressible fluids, Proc. Volta Congress, Rome, 1936.

\section{THE QUARTER-INFINITE WING OSCILLATING AT SUPERSONIC SPEEDS*}

By THEODORE R. GOODMAN (Cornell Aeronautical Laboratory, Inc.)

Introduction. In the April, 1951 issue of the Quarterly of Applied Mathematics, two papers appeared. One, by $\mathrm{H}$. J. Stewart and Ting-Yi $\mathrm{Li}^{1}$ purports to extend the method of J. C. Evvard ${ }^{2}$ for solving supersonic wing tips to the case of the oscillating wing. The other by J. W. Miles ${ }^{3}$ solves the problem of the oscillating rectangular airfoil by the Wiener-Hopf technique. In an application of their method to the rectangular airfoil, ${ }^{4}$ Stewart and Li obtain results which disagree with those of Miles. Moreover, Stewartson ${ }^{5}$ has treated the problem independently, and his results agree with Miles'.

The raison d'être of this note is to add yet another independent solution to the list of those which agree with Stewartson's and Miles'. In view of the fluxive state which this problem is in at the moment, it is felt that this would be of current interest.

The Gardner method. The method presented by C. S. Gardner ${ }^{6}$ will be used to obtain the solution. Let $x, y, z$ represent rectilinear space coordinates, $x$ in the direction of the free stream, and $y$ in the "spanwise" direction. Denote by $U$ the freestream velocity, $c$ the velocity of sound, $M$ the Mach number and $t$ the time. Let $z=f(x, y, t)$ describe the surface of the wing, $g=U f_{x}+f_{t}, \beta=\left(M^{2}-1\right)^{1 / 2}, x^{\prime}=x / \beta, t^{\prime}=\left(M x-\beta^{2} c t\right) / \beta$. Then in terms of $x^{\prime}, y, z, t^{\prime}$, Gardner's method for the rectangular airfoil consists in

*Received July 25, 1951.

${ }^{1}$ Stewart, H. J. and Li, Ting-Yi, Source-superposition method of solution of a periodically oscillating wing at supersonic speeds, Q. Appl. Math. 9, 31-45 (1951).

${ }^{2}$ Evvard, John C., Distribution of wave drag and lift in the vicinity of wing tips at supersonic speeds, NACA TN 1382, July 1947.

${ }^{3}$ Miles, John W., The oscillating rectangular airfoil at supersonic speeds, Q. Appl. Math. 9, 47-65 (1951). ${ }^{4}$ Stewart, H. J. and Li, Ting-Yi, Periodic motions of a rectangular wing at supersonic speeds, J. Aero.

Sci. 17, 529-538 (1951).

${ }^{5}$ Stewartson, K., On the linearized potential theory of unsteady supersonic motion, Q. J. Mech. and Appl. Math. 3, 182-199 (1950).

${ }^{6}$ Gardner, C., Time-dependent linearized supersonic flow past planar wings, Com. on Pure and Appl. Math., 3, 33-38 (1950). 
solving the following two boundary-value problems in succession: (The primes on $x^{\prime}$ and $t^{\prime}$ have been disregarded).

I) For $\xi \geq 0: \chi_{x x}-\chi_{t t}-\chi_{\xi \xi}=0$.

For $\xi=0: \chi=g(x, y, t)$.

For $x<0: \chi=0$.

II)

$$
\psi_{\xi \xi}-\psi_{\nu \nu}-\psi_{z z}=0 .
$$

For $y>0: \lim _{z \rightarrow 0+} \psi_{z}=\lim _{z \rightarrow 0-} \psi_{z}=\chi(\xi, x, y, t)$.

For $\xi>x: \psi \equiv 0$.

The velocity potential, $\varphi$, is determined by setting $\xi=0$ in the solution $\psi$.

The first problem. The value of $g$ is taken to be independent of $y$, and in terms of physical $x-t$ variables is given by

$$
g=v_{0}(x) \exp (i \omega t) .
$$

In terms of the transformed variables, this becomes

$$
g=v_{0}(x) \exp [i \nu(M x-t)]
$$

where

$$
\nu=\omega M / \beta U \text {. }
$$

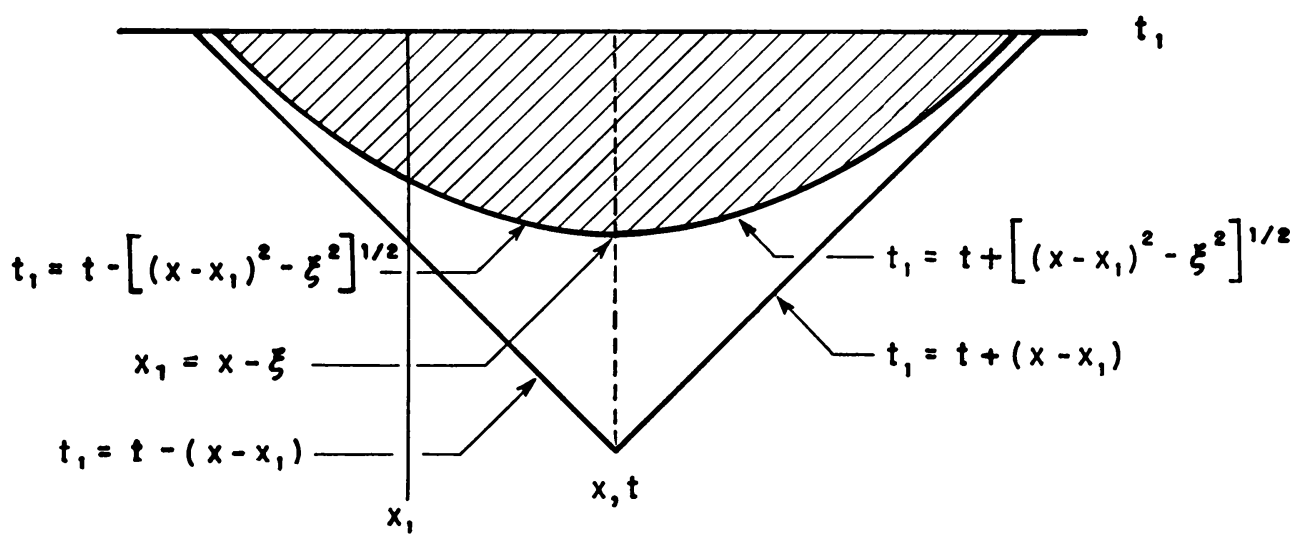

Fig. 1. "Wing" Problem in $x-t$ Space.

Let $\chi=\sigma_{\xi}$; then $\sigma$ may be determined by integrating sources over the shaded region shown in Fig. 1.

$$
\begin{aligned}
\sigma(\xi, x, t)=-\frac{1}{\pi} \int_{0}^{x-\xi} v_{0}\left(x_{1}\right) \exp \left(i \nu M x_{1}\right) d x_{1} \\
\cdot \int_{t-\left\{\left(x-x_{1}\right)^{2}-\xi^{2}\right]^{2 / 2}}^{t+\left[\left(x-x_{1}\right)^{2}-\xi^{2}\right]^{1 / 2}} \frac{\exp \left(-i \nu t_{1}\right) d t_{1}}{\left[\left(x-x_{1}\right)^{2}-\left(t-t_{1}\right)^{2}-\xi^{2}\right]^{1 / 2}} .
\end{aligned}
$$


If the substitution $t-t_{1}=\left[\left(x-x_{1}\right)^{2}-\xi^{2}\right]^{1 / 2} \cos \theta$ is made, the inner integral is recognized as a Bessel function. Then after some manipulation, Eq. (9) becomes:

$\sigma(\xi, x, t)=-\exp [i \nu(M x-t)] \int_{\xi}^{x} v_{0}(x-X) \exp (-i \nu M X) J_{0}\left[\nu\left(X^{2}-\xi^{2}\right)^{1 / 2}\right] d X$.

The second problem. The author has obtained elsewhere ${ }^{7}$ some general relationships which arise in using Gardner's method for the rectangular airfoil. In the two-dimensional region

$$
\varphi=-\sigma(0, x, t)
$$

Hence,

$$
\varphi=\exp [i \nu(M x-t)] \int_{0}^{x} v_{0}(x-X) \exp (-i \nu M X) J_{0}(\nu X) d X .
$$

In the tip region

$$
\varphi=-\sigma(0, x, t)+\pi^{-1} y^{1 / 2} \int_{y}^{x} \sigma(\xi, x, t)(\xi-y)^{-1 / 2} d \xi / \xi .
$$

Hence,

$$
\begin{aligned}
\varphi=\exp [i \nu(M x-t)] \int_{0}^{x} v_{0}( & -X) \exp (-i \nu M X) J_{0}(\nu X) d X \\
& -\pi^{-1} y^{1 / 2} \exp [i \nu(M x-t)] \int_{\nu}^{x}(\xi-y)^{-1 / 2} d \xi / \xi \\
& \cdot \int_{\xi}^{x} v_{0}(x-X) \exp (-i \nu M X) J_{0}\left[\nu\left(X^{2}-\xi^{2}\right)^{1 / 2}\right] d X .
\end{aligned}
$$

The wing and regions are depicted in Fig. 2.

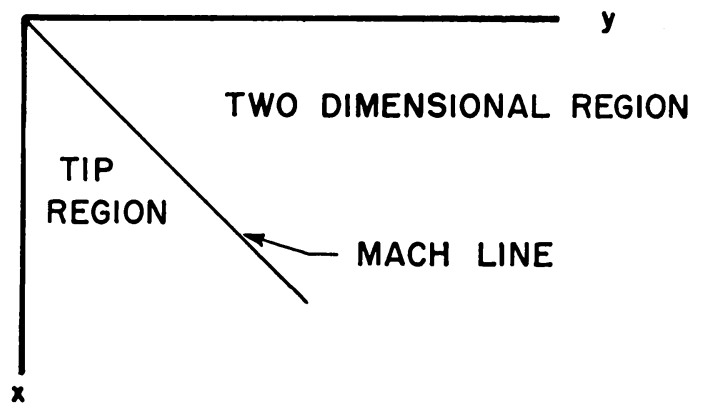

FIG. 2. Diagram of Wing

${ }^{7}$ Goodman, Theodore R., Aerodynamics of a supersonic rectangular wing striking a sharp-edged gust, J. Aero. Sci. 18, 519-526 (1951). 
The spanwise integral of the velocity potential. Let $\gamma$ represent the integral of $\varphi$ in the spanwise $(y)$ direction from the tip to the semispan $s$ :

$$
\begin{aligned}
\gamma=s \exp [i \nu(M x-t)] \int_{0}^{x} v_{0}(x-X) \exp (-i \nu M X) J_{0}(\nu X) d X \\
\quad-\pi^{-1} \exp [i \nu(M x-t)] \int_{0}^{x} y^{1 / 2} d y \int_{y}^{x}(\xi-y)^{-1 / 2} d \xi / \xi \\
\cdot \int_{\xi}^{x} v_{0}(x-X) \exp (-i \nu M X) J_{0}\left[\nu\left(X^{2}-\xi^{2}\right)^{1 / 2}\right] d X
\end{aligned}
$$

Consider only the triple integral appearing in the last term. Denote this by I. Upon interchanging the order of integration, there is obtained:

$I=\int_{0}^{x} d \xi \int_{\xi}^{x} v_{0}(x-X) \exp (-i \nu M X) J_{0}\left[\nu\left(X^{2}-\xi^{2}\right)^{1 / 2}\right] d X \int_{0}^{\xi}[y /(\xi-y)]^{1 / 2} d y$.

After integrating with respect to $y$, there is obtained:

$$
I=\pi / 2 \int_{0}^{x} d \xi \int_{\xi}^{x} v_{0}(x-X) \exp (-i \nu M X) J_{0}\left[\nu\left(X^{2}-\xi^{2}\right)^{1 / 2}\right] d X
$$

Interchanging the order of integration once again:

$$
I=\pi / 2 \int_{0}^{x} v_{0}(x-X) \exp (-i \nu M X) d X \int_{0}^{X} J_{0}\left[\nu\left(X^{2}-\xi^{2}\right)^{1 / 2}\right] d \xi .
$$

After letting $\xi=X \cos \theta$, the last integral will be recognized as a Struve function. ${ }^{8}$ Hence,

$$
I=\pi / 2 \int_{0}^{x} v_{0}(x-X) \exp (-i \nu M X) H_{-1 / 2}(\nu X)(\pi / 2 \nu X)^{1 / 2} X d X
$$

But $^{9}$

$$
H_{-1 / 2}(\nu X)=(2 / \pi \nu X)^{1 / 2} \sin \nu X
$$

Hence, finally there is obtained for $\gamma$ :

$\gamma=s \exp [i \nu(M x-t)] \int_{0}^{x} v_{0}(x-X) \exp (-i \nu M X)\left[J_{0}(\nu X)-(\sin \nu X) / 2 s \nu\right] d X$.

If account is taken for the difference in notation, it will be seen that this solution agrees with Miles' ${ }^{10}$ and Stewartson's. ${ }^{11}$

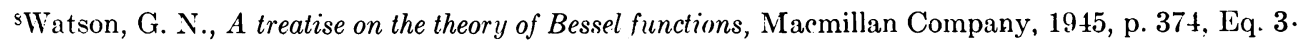

${ }^{9}$ ibid., pp. 328-338.

${ }^{10}$ loc. cit.

${ }^{11} l o c$. cit. 